A. Kaneko

Nagoya Math. J.

Vol. 104 (1986), 63-84

\title{
ON THE ANALYTICITY OF THE LOCUS OF SINGULARITY OF REAL ANALYTIC SOLUTIONS WITH MINIMAL DIMENSION
}

\author{
AKIRA KANEKO
}

\section{Introduction}

Let $P(x, D)$ be a linear partial differential operator with real analytic coefficients and let $C \subset \boldsymbol{R}^{n}$ be a germ of closed subset, say at the origin. We say that $C$ is (the locus of) an irremovable singularity of a real analytic solution $u$ of $P(x, D) u=0$ if $u$ is defined outside $C$ on a neighborhood $\Omega$ of 0 but cannot be extended to the whole neighborhood $\Omega$ even as a hyperfunction solution of $P(x, D) u=0$. This usage of the word "singularity" is the same as the one for the analytic functions in complex analysis, and is different of the usual usage of "singularities of solutions" in the theory of partial differential equations.

Until now we have given various results toward the determination of all the irremovable singularities of real analytic solutions. In this note we show that the minimal dimensional singularity $C$ contained in a noncharacteristic real analytic hypersurface $S$ is necessarily a real analytic submanifold. By taking the contraposition we simultaneously obtain a kind of continuation theorem for real analytic solutions. In our former work (Kaneko [3]) we could prove these results only under the additional condition that the solution $u$ is tempered near the singularity $C$. The proof below is based on a new result in complex analysis on continuation of holomorphic functions (see Theorem 1.7). The proof of the latter employs profound results of Nishino-Yamaguchi on a family of parabolic Riemann surfaces, and was communicated to me by Dr. Takeo Ohsawa [1]. I am very grateful to Dr. Ohsawa who allowed me to employ his proof in this note. I am indebted to all my colleagues of the department of mathematics for valuable discussions on the details of the proof, especially to Prof. K. Oikawa for his kind help for me to learn the necessary

Received February 13, 1985. 
knowledge from the theory of Riemann surfaces. I am also indebted to Prof. I. Wakabayashi and Prof. K. Kataoka for giving me precious comments on the careful reading of the manuscript.

Besides the above main result we discuss here on several related problems, e.g. on the timelike property of minimal dimensional irremovable singularities (Theorem 4.3) and on the construction of a real analytic solution with such a singularity (Theorem 4.4). These are more or less already treated by our earlier works but not so systematically.

\section{$\S 1$. Statement of results}

In order to explain the situation we repeat a part of our former work Kaneko [3]: Let $u$ be a real analytic solution of $P(x, D) u=0$ with the singularity $C$ contained in a non-characteristic hypersurface $S$. We can assume without loss of generality that $S=\left\{x_{1}=0\right\}$. Then $\left.u\right|_{\left\{ \pm x_{1}>0\right\}}$ are solutions of $P(x, D) u=0$ on $\left\{ \pm x_{1}>0\right\}$ respectively. Hence by the boundary value theory of Komatsu-Kawai-Schapira (see e.g. Komatsu-Kawai [1]), we can consider their hyperfunction boundary values $b_{j}^{ \pm}(u), 0 \leq j \leq m-1$ (where $m$ is the order of $P$ ) to $S$ from the respective sides. These are hyperfunctions of $x^{\prime}=\left(x_{2}, \cdots, x_{n}\right)$ defined as the uniquely determined coefficients of the expression

$$
P(x, D)[u]_{ \pm}= \pm \sum_{j=0}^{m-1} b_{j}^{ \pm}(u) \delta^{(m-j-1)}\left(x_{1}\right)
$$

where $[u]_{ \pm}$respectively denotes a uniquely determined extension of $\left.u\right|_{\left\{ \pm x_{1}>0\right\}}$ satisfying supp $[u]_{ \pm} \subset\left\{ \pm x_{1} \geq 0\right\}$ and an identity of the above form. Put

$$
b_{j}(u)=b_{j}^{+}(u)-b_{j}^{-}(u) \text {. }
$$

We have obviously

$$
\operatorname{supp} b_{j}(u) \subset C .
$$

The uniqueness of the boundary values implies especially the following

LEMMA 1.1. We have $b_{j}(u)=0,0 \leq j \leq m-1$ if and only if $C$ is a removable singularity.

To estimate S.S. (singular spectrum) of $b_{j}(u)$ we introduce the following sets (Kaneko [2]):

$$
\begin{aligned}
V_{S, A}^{ \pm}(P)=\{ & \left(x^{\prime}, \xi^{\prime}\right) \in S \times S^{n-2} ; \text { there exists a sequence }\left(x^{(k)}, \zeta^{(k)}\right) \text { such } \\
& \text { that } P_{m}\left(x^{(k)}, \zeta^{(k)}\right)=0, \pm x_{1}^{(k)}>0, \zeta^{\prime(k)} \in R^{n-1}, \pm \operatorname{Im} \zeta_{1}^{(k)}>0 \\
& \text { and that } \left.x^{(k)} \rightarrow\left(0, x^{\prime}\right), \zeta^{\prime(k)} \rightarrow \xi^{\prime}\right\}
\end{aligned}
$$


where $\xi^{\prime}=\left(\xi_{2}, \cdots, \xi_{n}\right)$ etc., and put

$$
V_{S, A}(P)=V_{S, A}^{+}(P) \cup V_{\bar{S}, A}(P) .
$$

These sets have coordinate invariant meanings: By an intrinsic expression $\left(x^{\prime}, \xi^{\prime}\right)$ stands for a point of the cosphere bundle of $S$, and $\xi_{1}^{(k)}$ stands for the roots of $P_{m}\left(x^{(k)}, \zeta_{1}^{(k)} \vartheta+\xi^{\prime}\right)=0$, where $\vartheta$ denotes the conormal of $S$ at $x^{\prime}$ to the positive side of $S$. In the case of an operator $P$ with constant coefficients (1.3) reduces to

$$
V_{S, A}(P)=S \times V_{(1,0, \ldots, 0) A}(P),
$$

where $V_{(1,0, \cdots, 0) A}(P)$ is the one introduced in Kaneko [1]:

$$
V_{(1,0, \cdots, 0) .1}(P)=\left\{\xi^{\prime} \in \boldsymbol{S}^{n-2} ; P_{m}\left(\zeta_{1}, \xi^{\prime}\right)=0 \text { has a non-real root for } \zeta_{1}\right\} .
$$

This is invariant by the linear coordinate transformations. By way of the regularity theorem up to the boundary (by Kaneko [2], Schapira [1], Kataoka [3]) we have S.S. $b_{J}^{ \pm}(u) \subset V_{S, A}^{ \pm}(P)$, hence

$$
\text { S.S. } b_{\jmath}(u) \subset V_{S, A}(P) \text {. }
$$

Definition 1.2. We say that $C$ is weakly timelike with respect to the operator $P$ if the conormal elements of $C$ as a subset of $S$ are all contained in $V_{S, A}(P)$.

If $C$ is not a submanifold of $S$, then we say that $\left(x^{\prime}, \xi^{\prime}\right)$ is a conormal element of $C$ if $x^{\prime} \in C$ and if by a local coordinate transformation of class $C^{1}$ in $S$ fixing $\left(x^{\prime}, \xi^{\prime}\right)$ the set $C$ comes in one side of the hyperplane with the conormal $\xi^{\prime}$ on a neighborhood of $x^{\prime}$. Remark that by this definition $\left(x^{\prime},-\xi^{\prime}\right)$ becomes a conormal element of $C$ if $\left(x^{\prime}, \xi^{\prime}\right)$ does.

By Kashiwara-Kawai's Holmgren type theorem (see e.g. Kaneko [6], appendix to Part II; see also Remark 2) after the proof of Theorem 1.5), the conormal elements of $\operatorname{supp} b_{j}(u)$ in this generalized sense must be contained in S.S. $b_{\jmath}(u)$. Thus from the estimates (1.1), (1.5) we obtain

Theorem 1.3. An irremovable singularity $C$ must be weakly timelike.

The dimension of a weakly timelike set $C$ is bounded from below by a constant depending on the operator $P$ and the hypersurface $S$. As is easily seen from the above theorem, this constant is given by the following

Definition 1.4. We let $r_{S}(P)$ denote the minimal value of the codimensions in $\boldsymbol{S}^{n-2}$ of concentric low-dimensional subspheres contained in 
the fiber of $V_{s, .1}(P)$ at the origin.

Here a concentric subsphere means the sphere analogue of the notion of a linear subspace.

Our main assertion in this note is the following

MaIn Theorem 1.5. Let $C$ be a germ of $C^{2}$-class submanifold of $S$ of dimension $r_{S}(P)$ at the origin. Assume that the fiber of $V_{S, A}(P)$ at the origin is invariant under the antipodal mapping $a: \xi^{\prime} \rightarrow-\xi^{\prime}$. Then if $C$ is an irremovable singularity of a real analytic solution $u$ of $P(x, D) u=0$, it must be automatically a germ of real analytic submanifold.

The essential step for this theorem is to prove the following lemma which is in itself interesting and will have many other applications:

Key Lemma 1.6. Let $v(x, t)$ be a germ of hyperfunction at the origin of $\boldsymbol{R}^{1+r}$ containing $t \in \boldsymbol{R}^{r}$ as real analytic parameters. Assume that there exists a germ of continuous function $\varphi(t)$ such that

$$
0 \in \operatorname{supp} v \subset C=\{x=\varphi(t)\} .
$$

Then $\varphi$ is in fact a germ of real analytic function.

Remark. The lemma remains true even if the hyperfunction part contains $m>1$ variables $x=\left(x_{1}, \cdots, x_{m}\right)$. This general case can be easily reduced to the above lemma by way of a definite integral with respect to a part of $x$ (see Kaneko [3], p. 3).

Reduction of Theorem 1.5 to Lemma 1.6. By Lemma 1.1 we have for some of $b_{j}(u)$

$$
0=\operatorname{supp} b_{j}(u) \subset C, \quad \text { S.S. } b_{j}(u) \subset V_{S, A}(P) .
$$

The definition of $r=r_{s}(P)$ implies that $b_{j}(u)$ contains at least $r$ coordinates as real analytic parameters (i.e. S.S. $b_{j}(u)$ does not contain the direction components which lie totally in the space of the dual variables of these coordinates). In fact, let $\gamma$ denote the $(n-2-r)$-dimensional suipsphere of the fiber $S^{n-2}$ of the cosphere bundle of $S$ at the origin, consisting of the conormal elements of $C$ in $S$ at 0 . By Kashiwara-Kawai's Holmgren type theorem we have $r \subset$ S.S. $\left.b_{j}(u)\right|_{x^{\prime}=0}$. We shall show that conversely S.S. $b_{i}(u)_{x^{\prime}=0} \subset \gamma$. Recall the watermelon theorem which asserts that a fiber of S.S. at 0 of a hyperfunction $u$ satisfying $0 \in \operatorname{supp} u \subset\left\{x_{1} \geq 0\right\}$ has the structure of the slice of a watermelon (see e.g. Kataoka [1], 
Theorem 4.3.3). Apply this to $b_{j}(u)$ with every pair of antipodal points in $\gamma$ as the north and the south poles. We see that if S.S. $\left.b_{j}(u)\right|_{x^{\prime}=0}$ contains some point outside $\gamma$, then it must contain a hemisphere of dimension $(n-$ $1-r)$ which is contained in the fibre of $V_{s, A}(P)$ at 0 by the above estimate. Since the latter set is invariant under the antipodal mapping $\xi^{\prime} \rightarrow-\xi^{\prime}$ by the assumption, we thus see that it contains in fact a subsphere of codimension $r-1$, which contradicts to the definition of $r=r_{S}(P)$.

Thus if we choose a system of coordinates in $S$ such that the dual variables of e.g. its last $r$ coordinates are transversal to the linear subspace spanned by $\gamma$, then $C$ takes the form as in Lemma 1.6, except that there are $n-r$ variables $x$. Together with the remark after that, this proves Theorem 1.5.

Remark. 1) The fibers of $V_{S, A}(P)$ are always invariant by the antipodal mapping if $P$ has constant coefficients because then $\left(V_{S, A}^{+}(P)\right)^{a}=$ $V_{\bar{S}, A}(P)$, but not in general if $P$ has variable coefficients. In Kanako [3] we forgot this assumption. Perhaps it is too strong as a condition which merely assures to apply Lemma 1.6. In fact, as is seen from the proof it suffices to assume that the fiber of $V_{S, A}(P)$ at the origin contains no closed hemisphere of codimension $r-1$.

2) We do not know for the present if we can replace the $C^{2}$-regularity of $C$ by the $C^{1}$-regularity in the above proof. A submanifold of class $C^{1}$ but not of $C^{2}$ cannot always be brought to one side of a hyperplane by a local analytic coordinate transformation. The Holmgren type theorem holds nevertheless for such submanifolds in view of the method of sweeping out (this remark is due to Dr. Ôaku). However we do not know if the watermelon theorem is valid for such a manifold.

3) As a matter of fact, even the assumption of $C^{1}$-regularity for $C$ is too strong. We can replace it by that of topological manifold in many cases. For example, such is the case if $r_{s}(P)=1$. In fact, we can then choose as $t$ any direction such that $\pm i d t \infty \notin V_{S, A}(P)$. The fact that $t=$ const. intersects $C$ always by only one point can be shown as follows: Assume that $\{t=c\} \cap C$ contains two points $p, q$. Then on the arc $\overparen{p q}$ of $C$ the coordinate $t$ must attain either its maximum or minimum value, and hence in view of Kashiwara-Kawai's Holmgren type theorem S.S. $b_{j}(u)$ must contain there the directions $\pm i d t \infty$, a contradiction.

In Kaneko [3] we proved the above Key lemma only for a distribution $v(x, t)$, which posed the restriction of temperedness to the solution 
near the singularity in the main result. There are several paraphrases for this lemma. (See Kaneko [8] where also a temporary improvement was given.) Here we give proof to the following variant:

Extended Hartogs Theorem 1.7. Let $F(z, \tau)$ be a holomorphic function in $1+r$ variables $(z, \tau)$ defined in

$$
\left(\{|z|<R\} \times\left\{\left|\tau_{1}\right|<1, \cdots,\left|\tau_{r}\right|<1\right\}\right) \backslash B,
$$

where $R>1$ and $B$ is a closed subset of $C^{1+r}$ satisfying the following properties:

1) The fiber $B_{\tau}=\{z \in C ;(z, \tau) \in B \cap(C \times\{\tau\})\}$ is contained in $|z| \leq 1$ for every $\tau$.

2) $B_{t}$ is a point $\{\varphi(t)\}$ for $\tau=t$ real.

Then $\varphi(t)$ becomes a real analytic function of $t$ unless $F$ is continued to the whole $B$.

The reduction of the Key lemma to Theorem 1.7 is as follows: In view of the real analytic parameters $t$ we can choose a set of defining functions $F_{ \pm}(z, \tau)$ of $v(x, t)$ such that

$$
v(x, t)=F_{+}((x, t)+i \Gamma 0)-F_{-}((x, t)-i \Gamma 0),
$$

where $\Gamma$ is a convex open cone containing the positive part of the imaginary $x$-axis. Since supp $v \subset C$, by way of the edge of the wedge theorem $F_{ \pm}(z, \tau)$ agree, through the real axis outside $C$, to a single holomorphic function $F(z, \tau)$. This function satisfies the assumption of Theorem 1.7 with the appropriate choice of coordinates.

\section{§2. Essential part of proof of Key lemma}

To prove Theorem 1.7 we reduce it to the original theorem of Hartogs [1] (see Hitotumatu [1], Theorem 3.14) which assures the same conclusion under the assumption that $B_{\tau}$ is a point even for every complex $\tau$. Thus we shall derive the latter situation from our assumption. To do so we can assume $r=1$. In fact, we can make the variables $\tau_{j}$ from real to complex successively for $j=1, \cdots, r$, with $\tau_{1}, \cdots, \tau_{j-1}$ fixed in the unit disc and $\tau_{j+1}, \cdots, \tau_{n}$ fixed in the real unit interval, and prove that $F(z, \tau)$ can beco ntinued up to $\{|z|<R\} \backslash\{\varphi(\tau)\}$ as a holomorphic function of $z$ for thus defined function $\varphi(\tau)$. Thus applying Hartogs' lemma (see BochnerMartin [1], Theorem 2 to Chapter VII or Hitotumatu [1], Lemma 3.11), we see that $F(z, \tau)$ becomes holomorphic outside the set $z=\varphi(\tau)$ (see also 
Remark 1) below). Further, by taking the negative power part of the Laurent expansion instead of $F(z, \tau)$ itself, we can assume that $R=+\infty$. Namely, we assume from the beginning that $F(z, \tau)$ is holomorphic in $(C \times$ $\{|\tau|<1\}) \backslash B$, where $B$ satisfies 1) and 2) of Theorem 1.7. The argument below is due to T. Ohsawa [1] as is mentioned in the introduction. (In fact in this situation we prove simultaneously that $\varphi(t)$ extends holomorphically to $\Delta$ ). To the convenience of the readers specialized in partial differential equations, we rather give detailed proofs to the facts which might be commonly known among geometers.

Let $\Delta$ denote the unit disc of $C$ and $\Delta^{R}$ the interval ]-1, 1[ of $R$. Let $D_{F}$ be the (maximal) domain of existence of the function $F(z, \tau)$ which is realized as a Riemann domain

$$
\pi: D_{F} \longrightarrow C \times \Delta
$$

over $C \times \Delta$. It contains an open subset $\Omega$ which corresponds homeomorphically to $(C \backslash \bar{A}) \times \Delta$ by $\pi$. We shall identify these in the sequel. Let $\rho$ : $C \times \Delta \rightarrow \Delta$ be the projection and consider the fibering

$$
\varpi=\rho \circ \pi: D_{F} \longrightarrow \Delta .
$$

For each fixed $\tau$, let $D$, denote the connected component of $\widetilde{\sigma}^{-1}(\tau)$ which contains $\Omega \cap \varpi^{-1}(\tau)$, and put

$$
D=\bigcup_{\tau \in \Delta} D_{*}
$$

This is obviously an open subset of $D_{F}$. (In fact, for $\tau_{0}$ fixed, any point $p \in D_{\tau_{0}}$ can be joined to a point of $\Omega \subset D_{\tau_{0}}$ by a path in $D_{\tau_{0}}$. Hence $D_{F^{\prime}}$ contains a neighborhood of this path each point of which can be joined to a point in $\Omega$ by a path in a fiber of $D_{F}$.) $D$ is a pseudo-convex Riemann domain as well as $D_{F}$, because we have removed from $D_{F}$ the union of (eventually uncountably many) complex submanifolds of codimension 1 . Note that $D$ is itself connected through $\Omega$. We shall denote the natural mappings $D \rightarrow C \times \Delta$ and $D \rightarrow \Delta$ by the same letters $\pi$ and $\varpi$ as above.

We next construct another Riemann domain $\tilde{D}$ whose fiber $\tilde{D}_{\tau}$ for each $\tau \in \Delta$ is the universal covering of $D_{\tau}$. First put

$$
\tilde{D}=\coprod_{\tau \in S} \tilde{D}_{\tau}
$$

as a set. The following is a variant of Hartogs' continuity theorem:

LEMMA 2.1. $\tilde{D}$ can be naturally endowed with the structure of a Riemann 
domain, with respect to which the natural mapping $\tilde{D} \rightarrow D$ induced from the mappings $\tilde{D}_{\tau} \rightarrow D_{\tau}$ is a local isomorphism.

Proof. It suffices to prove that we can give to $\tilde{D}$ a topology by which the set theoretical mapping $\tilde{D} \rightarrow D$ induced from the mappings $\tilde{D}_{\tau} \rightarrow D_{\tau}$ becomes a local homeomorphism. Fix $\tau_{0} \in \Delta$ arbitrarily. Since $D_{\tau_{0}}$ is an open Riemann surface, its fundamental group $\pi_{1}\left(D_{z_{0}}\right)$. is a free group with at most countably many free generators $\gamma_{1}, \gamma_{2}, \cdots$ (see e.g. Ahlfors-Sario [1], Chapter I, 44A and Chapter II, 12D). Without loss of generality, we can assume that $\gamma_{j}$ are closed curves of class $C^{1}$. In view of Lemma 2.2 below each $\gamma_{j}$ can be imbedded in a holomorphic family of curves $\gamma_{j}(\tau) \subset D_{\tau}$, $\left|\tau-\tau_{0}\right|<\varepsilon_{j}$.

For any positive integer $N$ not greater than the rank of $\pi_{1}\left(D_{\tau_{0}}\right)$, let $G_{N}$ denote the free subgroup of $\pi_{1}\left(D_{\tau_{0}}\right)$ generated by $\gamma_{1}, \cdots, \gamma_{N}$. When $\tau$ is sufficiently near $\tau_{0}$, the correspondence $\gamma_{j} \rightarrow \gamma_{j}(\tau), j=1, \cdots, N$ gives rise to a homomorphism of groups

$$
\varphi_{X}: G_{X} \longrightarrow \pi_{1}\left(D_{z}\right) .
$$

We shall show below that $\varphi_{N}$ is injective when $\tau$ is in a small neighborhood of $\tau_{0}$ (depending on $N$ ). Assuming this we can construct a neighborhood of each point $\tilde{p}$ of $\tilde{D}$ as a Riemann domain lying over $D$ as follows: Recall the standard method of construction of the universal covering. Choose any point $\tilde{p}$ of $\tilde{D}_{\tau_{0}}$ which lies over the point $p \in D_{\tau_{0}}$ after the round trip by an element $\gamma$ of $G_{N}$ for some $N$. Then there exists a small neighborhood $U$ of $p$ in $D$ such that to every point of $U \cap D_{\tau}$ there corresponds a unique point of $\tilde{D}_{\tau}$ which lies over it after the round trip by the image of $\gamma$ to $\pi_{1}\left(D_{\imath}\right)$. Let us employ the set $\tilde{U}$ of these points as a neighborhood of $\tilde{p}$ in $\tilde{D}$. The injectivity of $\varphi_{N}$ implies that $\tilde{U}$ 's for different $\tilde{p}$ 's over the same $p \in D$ are disjoint. Hence with this topology $\tilde{D}$ becomes a manifold which lies over $D$ naturally by the indicated mapping.

To show the injectivity of $\varphi_{N}$ it suffices to show the injectivity of its Abelianization

$$
\bar{\varphi}_{N}: G_{N} /\left[G_{N}, G_{N}\right] \longrightarrow \pi_{1}\left(D_{\tau}\right) /\left[\pi_{1}\left(D_{z}\right), \pi_{1}\left(D_{\tau}\right)\right] .
$$

In fact, $\pi_{1}\left(D_{\tau}\right)$ is likewise a free group by the above cited theorem. Therefore by the theorem of Nielsen-Schreier (see e.g. Kurosh [1], Chapter 9, $\S 35)$, Image $\varphi_{N}$ is also free unless it reduces to the unit element. The injectivity of $\bar{\varphi}_{N}$ implies that the rank of Image $\varphi_{N}$ is at least $N$. Since 
Image $\varphi_{N}$ is generated by $\varphi_{N}\left(\gamma_{1}\right), \cdots, \varphi_{N}\left(\gamma_{N}\right)$, these must be a set of free generators for Image $\varphi_{N}$ (see e.g. Kurosh [1], Chapter 9, $\S 39$ ). Thus $\varphi_{N}$ is injective.

Now by the Hurewicz theorem (see e.g. Ahlfors-Sario [1], Chapter I, 39D), $\pi_{1}\left(D_{\tau}\right) /\left[\pi_{1}\left(D_{\tau}\right), \pi_{1}\left(D_{\tau}\right)\right]$ is isomorphic to $H_{1}\left(D_{z}, Z\right)$. Hence the latter becomes a free Abelian group and we have $H_{1}\left(D_{v}, C\right) \cong H_{1}\left(D_{i}, Z\right) \otimes_{Z} C$. Therefore it suffices to show that $\gamma_{1}(\tau), \cdots, \gamma_{N}(\tau)$ define linearly independent elements of $H_{1}\left(D_{\imath}, C\right)$.

As a special case of the holomorphic de Rham theory for the Stein manifolds, there exist holomorphic 1 -forms $\omega_{1}, \cdots, \omega_{Y}$ on the open Riemann surface $D_{\tau_{0}}$ such that

$$
\int_{r_{j}} \omega_{k}=\delta_{j k}, \quad j, k=1, \cdots, N .
$$

Since $D$ is Stein, by Cartan's Theorem B for coherent $\mathcal{O}$-modules we can choose an extension of each $\omega_{k}$ to a holomorphic 1-form on $D$ (which we shall denote by the same letter). Then

$$
\int_{r_{j}(\tau)} \omega_{k}=\left.\int_{r_{j}(\tau)} \omega_{k}\right|_{D_{\tau}}, \quad j, k=1, \cdots, N
$$

are holomorphic functions of $\tau$ for $\left|\tau-\tau_{0}\right|<\min _{1 \leq j \leq x} \varepsilon_{j}$. The matrix with these entries has the determinant which is holomorphic in $\tau$ and is equal to 1 for $\tau=\tau_{0}$, hence different from 0 for $\tau$ sufficiently near $\tau_{0}$. Thus $\gamma_{j}(\tau)$, $j=1, \cdots, N$ are linearly independent in $H_{1}\left(D_{\imath}, C\right)$ for such $\tau$. This completes the proof of Lemma 2.1 mudulo the following

LEMMA 2.2. Let $\varpi: D \rightarrow \Delta$ be a surjective holomorphic mapping from a 2-dimensional Stein manifold to the unit disk. Let $\gamma$ be a mapping of class $C^{1}$ from the unit circle $\boldsymbol{S}^{1}$ to $D_{0}=\varpi^{-1}(0)$. If $\varpi$ has maximal rank for every point of $\gamma\left(\boldsymbol{S}^{1}\right)$, then there exist $\varepsilon>0$ and a mapping

$$
\Phi(\theta, \tau): \boldsymbol{S}^{1} \times\{|\tau|<\varepsilon\} \longrightarrow D
$$

of class $C^{1}$, holomorphic in $\tau$, such that

$$
\Phi(\theta, 0)=\gamma(\theta), \quad \Phi\left(S^{1}, \tau\right) \subset D_{s}=\widetilde{\sigma}^{-1}(\tau),
$$

Proof. We shall construct a holomorphic vector field $X$ on a neighborhood of the compact set $K=\gamma\left(\boldsymbol{S}^{1}\right)$ satisfying $\varpi_{*} X=d / d \tau$. $^{*}$ Then by

* In our present application we can simply adopt $X=\partial / \partial \tau$ since $D$ is a Riemann domain over $C \times \Delta$. 
integrating it we obtain a holomorphic mapping

$$
\Psi(z, \tau): K \times\{|\tau|<\varepsilon\} \longrightarrow D
$$

such that $\Psi(K, \tau) \subset D_{\tau}$ and that $\Psi(z, \tau): K \rightarrow D_{\tau}$ is an into homeomorphism for each fixed $\tau$. The required mapping can then be obtained by the composition $\Phi(\theta, \tau)=\Psi(\gamma(\theta), \tau)$. Note that $K$ is a Stein compact subset of $D$ contained in $D_{0}$ (in the sense that it admits a fundamental system of Stein neighborhoods).

On a neighborhood of every point of $K$ we can easily construct such a vector field $X_{\lambda}$ via a coordinate transformation to the product type. Thus we obtain a 0 -cochain $\left(X_{\lambda}\right)$ of vector fields on a neighborhood of $K$. Its coboundary $\left(X_{\lambda \mu}\right)$, where $X_{\lambda \mu}=X_{\mu}-X_{\lambda}$, satisfies $\widetilde{\varpi}_{*} X_{\lambda \mu}=0$. Since Ker $\widetilde{\varpi}_{*}$ is a coherent $\mathcal{O}$-module, by taking a refinement if necessary we can choose a $\mathcal{O}$-cochain $\left(Y_{\dot{\lambda}}\right)$ with values in $\operatorname{Ker} \widetilde{\sigma}_{*}$ satisfying $X_{\lambda \mu}=Y_{\mu}-Y_{\lambda}$, again by Cartan's Theorem B. Thus $X_{\lambda}-Y_{\lambda}$ 's glue together to a vector field $X$ globally defined on a neighborhood of $K$ and satisfying $\widetilde{\varpi}_{*} X=d / d \tau$. q.e.d.

Lemma 2.3. $\tilde{D}$ is a Stein Riemann domain.

This can be proven as in Nishino [2] based on Oka's pseudoconvexity theorem. Note that $\tilde{D}$ is not a true covering of $D$ in general. In fact, let $I I$ denote the natural mapping $\tilde{D} \rightarrow D$. From the proof of the above Lemma 2.1 we can easily see that a connected component $V_{k}$ of the inverse image $\Pi^{-1}(V)$ of a small neighborhood $V$ of a point $p \in D_{\tau_{0}}$ is homeomorphic to $V$ if it contains a point of $\Pi^{-1}(p)$, but this is not always the case. However, $V_{k}$ is always homeomorphic to $V \backslash \bigcup_{\tau \in T_{k}} D_{\tau}$ for some index set $T_{k}$, because each $\tilde{D}_{\tau}$ is a true covering of $D_{\tau}$, hence cannot have an end point in $V_{k}$. (Actually this dropping out of fibers results from the sudden vanishing of an element of $\pi_{1}\left(D_{\tau}\right)$ as $\tau$ approaches a fixed value $\tau \in T_{k}$. See examples in Remark 7) below.) Therefore let $\tilde{d}$ (resp. $d$ ) denote the distance from a point $p=\left(z_{0}, \tau_{0}\right)$ in $\tilde{D}$ (resp. $D$ ) up to the boundary measured by the equi-radius polydisc (i.e. supremum of the radius $r$ of a schlicht polydisc $\left\{\left|z-z_{0}\right|<r,\left|\tau-\tau_{0}\right|<r\right\}$ contained in $\tilde{D}$ (resp. D). By a theorem of Oka [1] (p. 222) it suffices to prove that $-\log \tilde{d}(p)$ is plurisubharmonic on $\tilde{D}$. With a constant $\varepsilon>0$, consider the function

$$
\tilde{f}_{\varepsilon}(p)=\max \{-\log \tilde{d}(p),-\log d(\Pi(p))+\varepsilon\} .
$$

This is plurisubharmonic on $\tilde{D}$. In fact, $-\log d(\Pi(p))$ is plurisubharmonic because $D$ is Stein and $\Pi$ is holomorphic. On the other hand, $-\log \tilde{d}(p)$ 
$\geq-\log d(\Pi(p))+\varepsilon$ implies that $\tilde{d}(p)<d(\Pi(p))$, that is, that in the connected component $V_{k}$ of $\Pi^{-1}(V)$, where $V$ is the polydisc of radius $d(\Pi(p))$ with center $\Pi(p)$, there exists an exceptional set as above such that $\tilde{d}(p)$ is equal to the distance from $p$ to it. Since this exceptional set is a union of submanifold of codimension $1,-\log \tilde{d}(p)$, hence $\tilde{f}_{s}(p)$ is plurisubharmonic on a neighborhood of such $p$. Because we have obviously $\tilde{d}(p) \leq d(\Pi(p))$, letting $\varepsilon \rightarrow 0$ we see that $-\log \tilde{d}(p)$ is plurisubharmonic.

Now we enter into the heart of the proof of Theorem 1.7:

Lemma 2.4. $D_{\tau}$ and $\tilde{D}_{\tau}$ are parabolic type Riemann surfaces for each $\tau \in \Delta$. (We only require the assertion for $\tilde{D}$ below.)

To show this we apply a theorem of Yamaguchi ([1], p. 84, Théorème; cf. also Nishino [3]). It suffices to verify the assumption of his theorem that $D_{\tau}$ (resp. $\tilde{D}_{\tau}$ ) is parabolic for $\tau$ belonging to a subset of $\Delta$ with positive logarithmic capacity. In our case this assumption is fulfilled for the subset $\Delta^{R}$, because for $t \in \Delta^{R}, D_{t}$ (resp. $\tilde{D}_{t}$ ) reduces to $C \backslash\{\varphi(t)\}$ (resp. its universal covering which is isomorphic to $C$ ). (The remaining assumption that $D$ (resp. $\tilde{D})$ contains a univalent subregion of the form $\{|z-b|<\delta\} \times \Delta$ is obviously satisfied by choosing a point corresponding to e.g. $z=2$ of the original domain and a small $\delta>0$.)

Thus each $\tilde{D}_{\tau}$ is conformally equivalent to the complex plane $C$. According to the famous type theorem for the universal coverings of Riemann surfaces (see e.g. Nevanlinna [1], VIII, 8.2), $D_{z}$ is conformally equivalent to either $C \backslash$ \{point\} or $C$ (because there is no possibility of the torus now).

Lemma 2.5. Each $D_{z}$ is conformally equivalent to $C \backslash\{$ point $\}$ unless $F(z, \tau)$ is holomorphic on $C \times \Delta$.

Proof. Assume that $D_{\tau_{0}} \approx C$ for some $\tau_{0}$. $D_{\tau_{0}}$ contains an open subset conformally equivalent to $C \backslash\{|z| \leq 1\}$ by the mapping $\pi_{\tau_{0}}=\left.\pi\right|_{\tau=\tau_{0}}: D_{\tau_{0}} \rightarrow C$.

The simple closed curve $\gamma$ in $D_{\tau_{0}}$ corresponding to the circle $|z|=2$ by this mapping contracts to a point in $D_{z_{0}}$. The image of this homotopy by $\pi_{\tau_{0}}$ gives the contraction of $|z|=2$ in $C$, which takes place in a bounded region $|z|<R$. Hence we see that the integral

$$
\frac{1}{2 \pi i} \oint_{|z|=R} \frac{F\left(\zeta, \tau_{0}\right)}{\zeta-z} d \zeta
$$

vanishes if $|z|>R$, by transporting it to the one on $D_{\tau_{0}}$ after deforming the path to $|z|=2$ and considering the above homotopy. Since this is 
true for $\tau$ sufficiently close to $\tau_{0}$, we can apply the standard method of proof of Hartogs' continuity theorem to conclude that $F(z, \tau)$ can be continued to a function holomorphic on the whole $C \times \Delta$ defined by the above integral but now with $z$ inside the circle $|z|=R$.

q.e.d.

Lemma 2.6. There exists a biholomorphic mapping $h(z, \tau): D \rightarrow(C \backslash\{0\})$ $\times \Delta$ preserving the fiber structure:

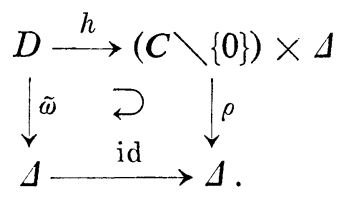

This follows from the uniformization theorem of Yamaguchi ([1], Théorème 3) combined with Hartogs' original theorem on the analyticity of the locus of singularity. This trivialization is already given in Nishino [1] when the fiber is isomorphic to $C$. We can deduce our assertion also from the latter by applying it to $\tilde{D}$ and going back to $D$ : Note that the discrete group of transformations of $C$ defining the covering map $\tilde{D}_{\tau} \rightarrow D_{\tau}$ admits a generator $T_{\imath}: w \rightarrow w+b(\tau)$ which is holomorphic in $\tau$, (where $w$ denotes the coordinate of the fiber $C$ in the trivialization $C \times \Delta$ of $\tilde{D}$ ). In fact, $C \times \Delta \approx \tilde{D}$ contains a sequence of holomorphic curves $w=w_{k}(\tau)$, $-\infty<k<\infty$ each of which is identified with $\{2\} \times \Delta$ in $(C \backslash\{|z| \leq 1\}) \times \Delta$, and hence $b(\tau)=w_{k+1}(\tau)-w_{k}(\tau)$. Therefore the analytic coordinate transformation $S:(w, \tau) \rightarrow\left(b(\tau)^{-1} w, \tau\right)$ of $C \times \Delta$ reduces $T_{\tau}$ to the translation $w \rightarrow w+1$ independent of $\tau$.

End of Proof of Theorem 1.7. Now we have a holomorphic mapping

$$
G(w, \tau):(C \backslash\{0\}) \times \Delta \approx D \longrightarrow C \times \Delta,
$$

which is univalent for $\tau=t \in \Delta^{R}$ with the image $C \backslash\{\varphi(t)\}$. This implies that for $\tau=t \in \Delta^{R}$ fixed the function $G(w, \tau)$ is holomorphic on $C \backslash\{0\}$ and bounded at $\{0\}$ in $w$. Hence by Riemann's theorem on removable singularity $G(w, \tau)$ extends to a function $\bar{G}(w, t)$ on the whole $C$. Consider the Laurent expansion of $G(w, \tau)$ with respect to $w$ at $w=0$. Then the coefficients are holomorphic functions of $\tau$ in the unit disc. By what is shown above the coefficients of the negative powers of $w$ vanish for $\tau \in \Delta^{R}$. Hence they vanish for all $\tau \in \Delta$. This means that $G(w, \tau)$ extends to a function $\bar{G}(w, \tau)$ holomorphic on the whole $C$ for every $\tau \in \Delta$. On the other hand, $G(w, \tau)$ has a polar singularity of order 1 along $w=\infty$ because $\pi: D \rightarrow C \times \Delta$ was 
an isomorphism on $\Omega$. Thus $G(w, \tau)$ reduces to a polynomial of degree 1 in $w$ and we conclude that $\bar{G}(w, \tau)$ is globally univalent in $w$ for each $\tau \in \Delta$, and defines an isomorphism $\bar{G}: C \times \Delta \rightarrow C \times \Delta$. This implies that $D$ was in fact a Euclidean region $\bar{G}((C \backslash\{0\}) \times \Delta)$ and $\varphi(t)=\bar{G}(0, t)$, that is, $\varphi(t)$ extends holomorphically to $\Delta$. This completes a fairly long proof of Theorem 1.7, hence of Lemma 1.6 and of Theorem 1.5.

Remark. 1) We cannot assume from the beginning that $t$ is one variable. This is because contrary to the holomorphic case a function $\varphi\left(t_{1}, \cdots\right.$, $t_{n}$ ), whose restriction to any real analytic curve is real analytic, need not be real analytic in $\left(t_{1}, \cdots, t_{n}\right)$ in general. Here is an example by M. Kashiwara: $\varphi\left(t_{1}, t_{2}\right)=\sqrt{ } t_{1}^{4}+t_{2}^{4}$. We know, however, that if $\varphi$ can be continued as a holomorphic function in each variable up to a disc of fixed radius, then $\varphi$ becomes holomorphic on a neighborhood of the real axis in all the variables (Theorem of Browder-Cameron-Storvick-Siciak; see Siciak [1] or Kajiwara [1], Theorem 3.4.1). We can apply this at the beginning of the proof of Theorem 1.7.

2) The use of the universal covering is inevitable, because there exist even plane domains which are parabolic but not conformally equivalent to $C$ or $C \backslash\{0\}$. See Ahlfors-Sario [1], Chapter IV, Theorem 22B and Lemma $24 \mathrm{~B}$.

3) The universal covering must be taken in the fiberwise way, because the universal covering of $D$ need not have simply connected fibers. Cf. example by $\mathrm{T}$. Terada: $D=(C \times \Delta) \backslash\left\{\tau=z^{2}\right\}$.

4) Lemma 2.1 is valid only for Stein manifolds of dimension 2 . Cf. example by T. Ohsawa: $D=\left(C^{2} \times \Delta\right) \backslash\left\{z_{1} z_{2}=\tau\right\}$.

5) Although $D$ lies after all in $C \times \Delta$, it seems difficult to show it directly: We must exclude such an example as the family of Riemann

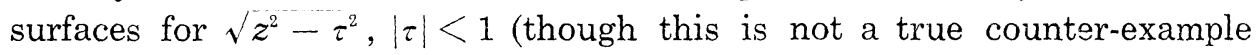
because the set $\{\tau=0\}$ is of capacity zero).

6) We cannot directly assert that $\tilde{D}_{\tau}$ is a universal covering of a plane region, neither: We must exclude such an example as

$$
f(z, \tau)=\int_{1}^{z} \exp \left(\tau / z^{2}\right) d z: C \backslash\{0\} \longrightarrow C \quad \text { (with } \tau \in \Delta \text { fixed) }
$$

For $\tau \neq 0$, this mapping is locally univalent because $(\partial f / \partial z)(z, \tau) \neq 0$, but does not define a covering map in the usual sense. Again in this example the set $\{\tau=0\}$ is of capacity zero. It seems to me that the general theory of parabolic coverings as in Chapter X of Tsuji [1] is helpless here. 
7) $\tilde{D}$ is not necessarily a true covering of $D$. Consider for example $D=(C \times \Delta) \backslash\left\{\tau=z^{2}\right\}$ in 3$)$. In this case our manifold $\tilde{D}$ lacks at $\tau=0$ countably many copies of $\tilde{D}_{0}$ as the limit of points of $\tilde{D}_{\tau}$ corresponding to the elements of $\pi_{1}\left(D_{\tau}\right)$ which are not in the image of elements of $\pi_{1}\left(D_{0}\right)$ by the mapping constructed in Lemma 2.1. If we could construct a true covering $\tilde{\tilde{D}}$ of $D$ by adding these lacking copies, then we would be able to apply a theorem of Stein-Nishino (see Stein [1] or Hitotumatu [1], p. 247) to conclude that $\tilde{\tilde{D}}$, hence $\tilde{D}$ is Stein. This is not true in general. For the

present example, such a manifold $\tilde{\tilde{D}}$ does not exist because it is impossible to define the natural covering mapping from the universal covering of $D$ whose fiber at the origin is not simply connected as mentioned above.

8) Note that the above proof contains the proof of Hartogs' original theorem as a special case: In that case by Hartogs' main theorem we can assume from the beginning that $\tau$ is a single variable. Then after the same reduction as above employing the Laurent expansion, we can apply the final part of the above proof to $D=(C \times \Delta) \backslash\{z=\varphi(\tau) ; \tau \in \Delta\}$. Note that the uniformization given in Lemma 2.6 is a very deep result. It seems difficult to extend Hartogs' original theorem directly to a general manifold with the fiber $C \backslash\{0\}$.

\section{§3. Examples and other applications}

To clarify the meaning of our result we first show the following example which we have often employed.

EXAMPLE 3.1. For the wave equation

$$
P(D)=D_{1}^{2}+\cdots+D_{n-1}^{2}-D_{n}^{2}
$$

with the time variable $x_{n}$, we have

$$
V_{S, A}(P)=\left\{\left(x^{\prime}, \xi^{\prime}\right) ; \xi_{2}^{2}+\cdots+\xi_{n-1}^{2} \geq \xi_{n}^{2}\right\},
$$

where $S=\left\{x_{1}=0\right\}$ and $x^{\prime}=\left(x_{2}, \cdots, x_{n}\right)$ etc. Hence $C \subset S$ is weakly timelike if and only if $C$ has no conormal element (i.e. supporting hyperplane) which is spacelike in the classical sense. Here we have $r_{s}(P)=1$, hence our main theorem asserts that if a curve $C \subset S$ is an irremovable singularity for a real analytic solution of the wave equation, then it must be a weakly timelike real analytic curve. (Note that for this example the conclusion of weak timelikeness is rather classical, but that of analyticity is not at all classically obvious.) For this situation we can further assert 
that $C$ must be either a timelike real analytic curve in the usual sense (i.e. such that its tangent vectors are timelike) or a bicharacteristic (hence non-timelike) straight line contained in $S$. See the note added in proof of Kaneko [5] concerning this. Conversely every such $C$ can become in fact an irremovable singularity of some real analytic solution of the wave equation. In the next section we shall discuss about these related problems in a little more generalized situation.

A more interesting (and less classical) example can be obtained if we consider the ultra-hyperbolic equation

$$
P(D)=D_{1}^{2}+\cdots+D_{k}^{2}-D_{k+1}^{2}-\cdots-D_{n}^{2}, \quad 2 \leq k \leq n-2 .
$$

Here the number $r_{S}(P)$ varies according to the choice of $S: r_{S}(P)=n-$ $k$ resp. $k$ according to $S=\left\{x_{1}=0\right\}$ resp. $\left\{x_{k+1}=0\right\}$. For this operator we do not know if the alternative between timelike and non-timelike property holds.

Although the conclusion of Theorem 1.3 is best possible as a joint estimate for all the real analytic solutions of an operator, there exist some more delicate cases where we can apply Lemma 1.6 to individual real analytic solutions. For example, for a real analytic solution $u$ of the partial Laplace equation

$$
P(D)=D_{1}^{2}+\cdots+D_{k}^{2} \text { on } R^{n} \quad \text { with } n>k,
$$

or of the Cauchy-Riemann equation

$$
P(D)=D_{1}+i D_{2} \quad \text { on } R^{n} \quad \text { with } \quad n>k=2,
$$

we can assert that the difference of boundary values $b_{j}(u)=b_{j}^{+}(u)-b_{j}^{-}(u)$ to $x_{1}=0$ contains $x_{k+1}, \cdots, x_{n}$ as real analytic parameters (though we cannot estimate S.S. $b_{j}(u)$ by a closed set independent of $u$ which is disjoint with the set $\left.\boldsymbol{R}^{n} \times\left\{i \boldsymbol{R} d x_{k+1} \infty+\cdots+i \boldsymbol{R} d x_{n} \infty\right\}\right)$. See Schapira [2], Kaneko [5]. Thus we can apply our argument to obtain the following theorem. (Such a result seems not at all obvious from the viewpoint of complex analysis.)

THEOREM 3.2. Let $u(x, t)$ be a real analytic function defined on a neighborhood of $0 \in \boldsymbol{R}^{n+m}$ outside the topological submanifold

$$
C: \quad x_{1}=0, x_{j}=\varphi_{j}(t), 2 \leq j \leq n .
$$

Assume further that $u$ is there harmonic in $x$ (or complex holomorphic in 
$x_{1}+i x_{2}$ with $\left.n=2\right)$. Then $C$ must be a germ of real analytic submanifold at 0 .

We knew before that if $u(x, t)$ is harmonic in a proper subset of the variables $x$ (or if $n>2$ in the latter case), that is, if $C$ has dimension lower than the reasonable value, then $u(x, t)$ becomes in fact real analytic on the whole neighborhood of 0 . (Cf. the proof of Theorem 1.3 combined with the above quoted result on micro-analyticity of the boundary values.) Theorem 3.2 asserts that $C$ is removable even if it has the reasonable dimension unless it is real analytic.

\section{$\S 4$. Timelike property of the singularity}

In many cases the minimal dimensional irremovable singularities have a little more restricted type. To describe this we recall here the definition of timelikeness given in Kaneko [4]. Assume again that $S=\left\{x_{1}=0\right\}$ is non-characteristic with respect to $P$. We put

$$
V_{S, A}^{0}(P)=\left\{\left(x^{\prime}, \xi^{\prime}\right) \in S \times \boldsymbol{S}^{n-2} ; P_{m}\left(0, x^{\prime}, \xi_{1}, \xi^{\prime}\right) \neq 0 \text { if } \xi_{1} \in \boldsymbol{R}\right\} .
$$

We have $V_{S, A}^{0}(P) \subset V_{S, A}(P)$. In fact, $P_{m}\left(0, x^{\prime}, \xi_{1}, \xi^{\prime}\right) \neq 0$ for all $\xi_{1} \in \boldsymbol{R}$ implies that the equation $P_{m}\left(0, x^{\prime}, \zeta_{1}, \xi^{\prime}\right)=0$ has complex roots. Hence by the continuity of the roots $\left(x^{\prime}, \xi^{\prime}\right)$ must belong to at least either of $V_{S, A}^{ \pm}(P)$ according to the sign of the imaginary part of the complex roots.

Definition 4.1. We say that a subset $C$ of $S$ is timelike with respect to $P$ if the conormal elements of $C$ as a subset of $S$ are contained in $V_{S, A}^{0}(P)$.

These definitions have invariant meanings for a general real analytic non-characteristic hypersurface $S$. We further remark that our definition of timelikeness is coherent with the general one given by F. John [1]:

Definition $4.1^{\prime}$. A set $C \subset \boldsymbol{R}^{n}$ is called timelike with respect to $P$ if every conormal element of $C$ as a subset of $\boldsymbol{R}^{n}$ is non-characteristic with respect to $P$.

In fact, a conormal element $\left(0, x^{\prime}, \xi_{1}, \xi^{\prime}\right)$ of $C$ in $\boldsymbol{R}^{n}$ just comes from the conormal element $\left(x^{\prime}, \xi^{\prime}\right)$ of $C$ in $S$. The terminology of timelikeness of course comes as an analogy from the case of the wave equation. See Example 3.1 above.

We guess that in general an irremovable singularity $C$ of minimal dimension (hence analytic) is not only weakly timelike but is alternatively either timelike or everywhere non-timelike, that is, the conormal bundle 
of $C$ is either totally contained in $V_{S, A}^{0}(P)$ or always contains a point of $V_{S, A}(P) \backslash V_{S, A}^{0}(P)$ in each fiber. Here we shall prove this for some class of operators with constant coefficients.

Definition 4.2. We shall say that $P(x, D)$ has regular timelike region with respect to $S$ if $V_{S, A}^{0}(P)$ agrees with the interior of $V_{S, A}(P)$ as the subset of the cosphere bundle of $S$.

THEOREM 4.3. Let $P(D)$ be an operator with constant coefficients. Assume that $S=\left\{x_{1}=0\right\}$ is non-characteristic with respect to $P, P$ has regular timelike region with respect to $S$, and that $r_{S}(P)=1$. Then a minimal dimensional irremovable singularity $C$ of a real analytic solution $u$ of $P(D) u$ $=0$ contained in $S$ is either everywhere timelike or everywhere non-timelike.

Proof. Assume that $C$ possesses a conormal element, say $\left(0, \nu^{\prime}\right)$, contained in $V_{S, A}(P) \backslash V_{S, A}^{0}(P)$. The assumption that $P$ has regular timelike region implies that the equation $P_{m}\left(\zeta_{1}, \nu^{\prime}\right)=0$ for $\zeta_{1}$ has only real roots, in view of the continuity of the roots of an algebraic equation. Therefore by a result on the propagation of micro-analyticity along the boundary (Kaneko [7], Theorem 2.5), we see that $C \cap\left\{x^{\prime} \nu^{\prime}=0\right\}$ cannot contain a compact connected component. Since $C$ is a real analytic curve in view of Theorem 1.5 and Remark after that, it must be identically contained in $x^{\prime} \nu^{\prime}=0$. Thus $C$ contains the direction $\nu$ erywhere in its conormal elements, hence everywhere non-timelike.

q.e.d.

Finally as a partial converse of the above arguments we give proof to the following theorem briefly sketched in Kaneko [4]:

THEOREM 4.4. Every germ of timelike real analytic submanifold $C$ becomes an irremovable singularity of a real analytic solution of $P(x, D) u=0$.

Proof. First note that a timelike real analytic submanifold $C$ can be contained (at least locally) in a real analytic hypersurface $S$ non-characteristic with respect to $P$. Hence we can assume without loss of generality that $C$ is contained in the hyperplane $S=\left\{x_{1}=0\right\}$ which is non-characteristic with respect to $P$. Let $\delta_{C}\left(x^{\prime}\right)$ denote the measure on $S$ with support in $C$ which defines the curvilinear integration along $C$ as a distribution. Then the product $\delta_{C}(x)=\delta_{C}\left(x^{\prime}\right) \delta\left(x_{1}\right)$ is a distribution corresponding to the curvilinear integration along $C$ in $\boldsymbol{R}^{n}$. We have

$$
\text { S.S. } \delta_{C}(x)=\boldsymbol{S}_{C}^{*} \boldsymbol{R}^{n} \quad(\text { the conormal elements of } C) \text {. }
$$


By the assumption this set is contained in $V_{S, A}^{0}(P)$, hence $P$ is microelliptic on this set. Thus we can obtain a well defined microfunction $P^{-1} \delta_{c}(x)$ with support in this set. Let $v(x)$ be a hyperfunction representative of this microfunction. Then we have

$$
P(x, D) v=\delta_{C}(x)+h,
$$

where $h$ is a germ of real analytic function. Choose a real analytic solution $w$ of $P(x, D) w=h$ and put $u=v-w$. Then we obtain a solution $u$ of

$$
P(x, D) u=\delta_{c}(x)=\delta_{c}\left(x^{\prime}\right) \delta\left(x_{1}\right)
$$

such that S.S. $u \subset \boldsymbol{S}_{C}^{*} \boldsymbol{R}^{n}$. Thus $u$ is a real analytic solution of $P(x, D) u$ $=0$ outside $C$. The fact that $C$ is an irremovable singularity of $u$ can be shown as follows: By the uniqueness of the boundary values the identity (4.5) implies that

$$
b_{m-1}^{+}(u)-b_{m-1}^{-}(u)=\delta_{C}\left(x^{\prime}\right) \neq 0,
$$

hence in view of Lemma $1.1 C$ is irremovable.

q.e.d.

ExAmple 3.1 (continued). In view of the above theorem the wave equation

$$
P(D) u=\left(D_{1}^{2}+\cdots+D_{n-1}^{2}-D_{n}^{2}\right) u=0
$$

possesses a real analytic solution $u$ with any prescribed timelike real analytic curve $x^{\prime}=\varphi\left(x_{n}\right)$ as irremovable singularity. Since the timelike/nontimelike alternative holds, the remaining possibility of irremovable singularity $C$ contained in $S=\left\{x_{1}=0\right\}$ is only that of the bicharacteristic lines. It is well known that the above equation possesses a real analytic solution $u$ whose singular support agrees with a bicharacteristic line (see e.g. Kawai [1]). However this singularity is of course removable as a hyperfunction solution in our sense. We shall here show that there exists in fact a real analytic solution $u$ which has a bicharacteristic line as irremovable singularity:*) Let $E\left(x_{1}, \cdots, x_{n-2}\right)$ be a fundamental solution of the Laplace equation: $\left(D_{1}^{2}+\cdots+D_{n-2}^{2}\right) E=\delta\left(x_{1}, \cdots, x_{n-2}\right)$. (We assume $n \geq 3$ because otherwise the problem is trivial.) Then the hyperfunction

$$
v(x)=E\left(x_{1}, \cdots, x_{n-2}\right) \delta\left(x_{n-1}-x_{n}\right)
$$

satisfies

*) The author is much obliged to the referee who kindly indicated this method of construction. 


$$
P(D) v=\delta\left(x_{1}, \cdots, x_{n-2}\right) \delta\left(x_{n-1}-x_{n}\right) .
$$

Hence $v$ is a hyperfunction solution with the bicharacteristic line

$$
x_{1}=\cdots=x_{n-2}=0, \quad x_{n-1}=x_{n}
$$

as irremovable singularity. We shall modify this to a real analytic one. Note that the initial data

$$
\begin{aligned}
& \left.v\right|_{x_{n}=0}=E\left(x_{1}, \cdots, x_{n-2}\right) \delta\left(x_{n-1}\right), \\
& \left.\frac{\partial v}{\partial x}\right|_{x_{n}=0}=-E\left(x_{1}, \cdots, x_{n-2}\right) \delta^{\prime}\left(x_{n-1}\right)
\end{aligned}
$$

have the following estimate for S.S.:

$$
\text { S.S. }\left(\left.\frac{\partial^{j} U}{\partial x_{n}^{j}}\right|_{x_{n}=0}\right) \subset\left(\left\{x_{n-1}=0\right\} \times\left\{ \pm i d x_{n-1} \infty\right\}\right) \cup\left(\left\{x_{1}=\cdots=x_{n-2}=0\right\} \times S^{n-2}\right) .
$$

Let $f_{j}\left(x_{1}, \cdots, x_{n-1}\right), j=0,1$ be hyperfunctions of $n-1$ variables such that

$$
\frac{\partial^{j} v}{\partial x_{n}^{j}} x_{n=0}-f_{j}\left(x_{1}, \cdots, x_{n-1}\right), j=0,1 \text { are real analytic outside }
$$
the origin of $\boldsymbol{R}^{n-1}$;

The existence of such $f_{j}$ is assured in view of the above estimate for S.S. $\left(\partial^{j} v /\left.\partial x_{n}^{j}\right|_{x_{n}=0}\right)$ and the flabbiness of the sheaf $\mathscr{C}$ of microfunctions. Then let $w$ be the solution of the Cauchy problem

$$
\left\{\begin{array}{l}
P(D) w=0 \\
\partial^{j} w \\
\hdashline \partial x_{n}^{j} x_{x_{n}=0}=f_{j}, \quad j=0,1 .
\end{array}\right.
$$

In view of the estimate (4.8) we have

$$
\text { sing supp } w \subset\left\{x_{n-1}^{2}=x_{n}^{2}\right\} .
$$

Put $u=v-w$. Then $u$ satisfies

$$
\left\{\begin{array}{l}
P(D) u=\delta\left(x_{1}, \cdots, x_{n-2}\right) \delta\left(x_{n-1}-x_{n}\right), \\
\left.\frac{\partial^{j} u}{\partial x_{n}^{j}}\right|_{x_{n}=0}=\left.\frac{\partial^{j} v}{\partial x_{n}^{j}}\right|_{x_{n}=0}-f_{j}, \quad j=0,1 .
\end{array}\right.
$$

In view of (4.7) we see from this equation that

$$
\text { sing supp } u \subset\left\{x_{n}^{2} \geq x_{1}^{2}+\cdots+x_{n-1}^{2}\right\} \text {. }
$$


Since we have on the other hand

$$
\operatorname{sing} \operatorname{supp} u \subset \operatorname{sing} \operatorname{supp} v \cup \operatorname{sing} \operatorname{supp} w \subset\left\{x_{n-1}^{2}=x_{n}^{2}\right\},
$$

hence by taking the intersection we conclude that

$$
\text { sing supp } u \subset\left\{x_{1}=\cdots=x_{n-2}=0, x_{n-1}^{2}=x_{n}^{2}\right\} .
$$

Thus the solution $u$ considered on a neighborhood of points on the bicharacteristic line (4.6) except for the origin will serve as a required example.

Remark. 1) The minimal dimension of the singularity constructible by the above theorem is given by the number $r_{S}^{0}(P)$ defined as the minimal value of the codimensions in $\boldsymbol{S}^{n-2}$ of concentric subspheres contained in the fiber of $V_{S, A}^{0}(P)$ at the origin. The construction of singularity with higher dimension is much more free because we can use the superposition.

2) To extend these studies to the region $V_{S, A}(P) \backslash V_{S, A}^{0}(P)$ when it is not thin, we must prepare a kind of factorization theory of the operator which should lie between the one in the sense of differential polynomials and that of pseudo-differential operators. For the moment we know the definitive answer only in the case of singularities of dimension 0 (i.e. isolated points) even for operators with constant coefficients: $P(D)$ has a real analytic solution with an isolated singularity if and only if $P(D)$ has an elliptic factor $Q(D)$ in the sense of polynomials (for which we have obviously $\left.r_{S}^{0}(Q)=0\right)$. For more about this problem see Part III of Kaneko [6].

The problem of analyticity of the singularities of real analytic solutions is proposed by Prof. K. Aomoto on the occasion of the oral examination for my master thesis which contains the above cited result on real analytic solutions with isolated singularities. We believe that in this note we have made an important progress to this problem. Nevertheless it remains to treat singularities which are not contained in a real analytic non-characteristic hypersurface. Note that a timelike submanifold $C$ is contained in a non-characteristic hypersurface, but only of the same regularity as $C$ : We cannot assume in general that this hypersurface may be chosen real analytic from the beginning. For the moment we do not know any tool to treat this general case. It is possible that there appears an analytic set with singularities as the locus of such a general singularity. 


\section{REFERENCES}

Ahlfors L. \& Sario L. [1] Riemann Surfaces, Princeton Univ. Press, Princeton, 1960. Bochner S. \& Martin W. T. [1] Several Complex Variables, Princeton Univ. Press, Princeton, 1948.

Hartogs F. [1] Über die aus den singulären Stellen einer analytischen Funktion mehrerer Veränderlichen bestehenden Gebilde, Acta Math., 32 (1908), 57-79.

Hitotumatu S. [1] Theory of Analytic Functions of Several Variables (in Japanese), Baifûkan, Tokyo, 1960.

John F. [1] Plane Waves and Spherical Means, Interscience, New York, 1955.

Kajiwara J. [1] Theory of Complex Functions (in Japanese), Morikita, Tokyo, 1968. Kaneko A. [1] On the singular spectrum of boundary values of real analytic solutions, J. Math. Soc. Japan, 29 (1977), 385-398.

[2] Singular spectrum of boundary values of solutions of partial differential equations with real analytic coefficients, Sci. Papers College Gen. Ed. Univ. Tokyo, 25 (1975), 59-68; Correction in Appendix B of Kaneko [5].

[3] Analyticity of minimal dimensional singularity of real analytic solutions, ibid., 26 (1976), 1-5.

[4] On continuation of regular solutions of linear partial differential equations, Publ. RIMS Kyoto Univ., 12, Suppl. (1977), 113-121.

[5] Estimation of singular spectrum of boundary values for some semihyperbolic operators, J. Fac. Sci. Univ. Tokyo Sec. 1A, 27 (1980), 401461.

[6] On continuation of real analytic solutions of linear partial differential equations, Astérisque, 89-90, Soc. Math. France, 1981, pp. 11-44.

[7] On the propagation of micro-analyticity along the boundary, J. Fac. Sci. Univ. Tokyo Sec. 1A, 29 (1982), 319-352.

[8] On the analyticity of the locus of singularity of real analytic solutions with minimal dimension (in Japanese), Sûrikaiseki-kenkyûsho Kôkyûroku, 533 (1984), 256-271.

Kataoka K. [1] On the theory of Radon transformations of hyperfunctions, J. Fac. Sci. Univ. Tokyo Sec. 1A, 28 (1981), 331-413.

[2] Micro-local theory of boundary value problems I, J. Fac. Sci. Univ. Tokyo Sec. 1A, 27 (1980), 355-399.

[3] Micro-local theory of boundary value problems II, J. Fac. Sci. Univ. Tokyo Sec. 1A, 28 (1981), 31-56.

Kawai T. [1] Construction of elementary solutions for $I$-hyperbolic operators and solutions with small singularities, Proc. Japan Acad., 46 (1970), 912-916.

Komatsu H. \& Kawai. T. [1] Boundary values of hyperfunction solutions of linear partial differential equations, Publ. RIMS Kyoto Univ., 7 (1971), 95-104.

Kurosh A. G. [1] Theory of Groups I, II, Chelsea, New York, 1955-56.

Nevanlinna R. [1] Uniformisierung, Springer, Berlin-Göttingen-Heidelberg, 1953.

Nishino T. [1] Nouvelles recherches sur les fonctions entières de plusieurs variables complexes (II). Fonctions entières qui se reduisent à celles d'une variable, J. Math. Kyoto Univ., 9 (1969), 221-274.

[2] Ditto (III). Sur quelques propriétés topologiques des surfaces premières,ibid., 10 (1970), 245-271.

[3] Ditto (IV). Types de surfaces premières, ibid., 13 (1973), 217-272.

Ohsawa T. [1] Personal correspondences, September 1984 to January 1985. 
Oka K. [1] Sur les fonctions analytiques de plusieurs variables IX. Domaines finis sans point critique intérieur, Japan. J. Math., 27 (1953), 97-155; Reprinted in Collected Papers from Iwanami, Tokyo 1961.

Schapira P. [1] Propagation au bord et réflexion des singularités analytiques des solutions des équations aux dérivées partielles, Séminaire GoulaouicSchwartz 1975-76, exposé 6.

[2] Propagation au bord et réflexion des singularités analytiques des solutions des équations aux dérivées partielles II, Séminaire GoulaouicSchwartz 1976-77, exposé 9.

Siciak J. [1] Analyticity and separate analyticity of functions defined on lower dimensional subsets of $C^{n}$, Zeszyty Nauk. Uniw. Jagielloń, Prace Mat., 13 (1969), 53-70.

Stein K. [1] Überlagerungen holomorph-vollständiger komplexer Räume, Arch. Math., 7 (1956), 354-361.

Tsuji M. [1] Potential Theory, Maruzen, Tokyo, 1959.

Yamaguchi H. [1] Parabolicité d'une fonction entière, J. Math. Kyoto Univ., 16 (1976), 71-92.

Department of Mathematics

College of General Education

University of Tokyo

3-8-1, Komaba, Meguro-ku

Tokyo 153, Japan 